\title{
Pore-Scale Investigation of Biomass Plug Development and Propagation in Porous Media
}

\author{
Terri L. Stewart, ${ }^{*}$ H. Scott Fogler \\ Department of Chemical Engineering, University of Michigan, \\ 3168 H.H. Dow Building, Ann Arbor, Michigan 48109-2136, USA; \\ telephone: (734)-763-1361; fax: (734)-763-0459; \\ e-mail: sfogler@engin.umich.edu
}

Received 12 March 2001; accepted 26 June 2001

\begin{abstract}
Biomass plugging of porous media finds application in enhanced oil recovery and bioremediation. An understanding of biomass plugging of porous media was sought by using a porous glass micromodel through which biomass and nutrient were passed. This study describes the pore-scale physics of biomass plug propagation of Leuconostoc mesenteroides under nutrient-rich conditions. It was found that, as the nutrient flowed through the micromodel, the initial biomass plug occurred at the nutrient--inoculum interface due to growth in the larger pore throats. As growth proceeded, biomass filled and closed these larger pore throats, until only isolated groupings of pore throats with smaller radii remained empty. As nutrient flow continued, a maximum pressure drop was reached. At the maximum pressure drop, the biomass yielded in a manner similar to a Bingham plastic to form a breakthrough channel consisting of a path of interconnected pore throats. The channel incorporated the isolated groupings of empty pore throats that had been present before breakthrough. As the nutrient flow continued, subsequent plugs developed as breakthrough channels refilled with biomass and in situ growth was stimulated in the region just downstream of the previous plug. The downstream plugs had a higher fraction of isolated groupings of empty pore throats, which can be attributed to depletion of nutrient downstream. When the next breakthrough channel formed, it incorporated these isolated groupings, causing the breakthrough channels to be branched. It was observed that the newly formed plug could be less stable with this higher fraction of empty pore throats and that the location of breakthrough channels changed in subsequent plugs. This change in breakthrough channel location could be attributed to the redistribution of nutrient flow and the changes in flowrate in the pore throats. (c) 2002 John Wiley \& Sons, Inc. Biotechnol Bioeng 77: 577-588, 2002; DOI 10.1002/bit. 10044
\end{abstract}

Keywords: Leuconostoc mesenteroides; biomass plugging; porous media; pore scale

\section{INTRODUCTION}

Biomass plugging of porous media has great potential in enhanced oil recovery. Using bacterial profile modifi-

Correspondence to: H.S. Fogler

* Present address: Pacific Northwest National Laboratory, P.O. Box 999, MSIN K9-2, Richland, WA 99352 cation (BPM) - a process in which the highly permeable, watered-out regions of oil reservoirs are plugged with bacterial cells and exopolymers produced by the cells (i.e., biomass) - residual oil can be recovered. In this approach, bacteria and nutrient solution are injected into the reservoir. The system is shut-in to allow in situ biomass growth that develops into a biomass plug in the more permeable region. Subsequently, water is injected to force oil trapped in less permeable regions of the reservoir to be pushed out into the recovery well (i.e., water flooding). A similar scheme has been envisioned for groundwater remediation. In this application, biomass would be developed in situ to create a subsurface biobarrier that diverts clean groundwater around a contaminated zone, thus isolating a waste site.

For BPM or similar applications to be employed successfully, three factors must be considered. First, the bacterial cells must be placed throughout the entire watered-out zone during inoculation of the soils, because the inoculum serves as the basis for a biomass plug. By using starved bacterial cells, the cell dimension is greatly reduced, improving its dispersion into the porous media. In addition, the use of bacteria whose exopolymer production can be switched on or off is of particular interest in BPM, because it is the exopolymer production that is key to fluid transport and plugging of the porous media. The production of exopolymers can be switched off to encourage deep penetration of bacteria during inoculation and flow through the porous media and then switched on for in situ growth and exopolymer production to establish a biomass plug. Leuconostoc mesenteroides (strain NRRL B523), a bacterial species found in the natural environment, has a reduced diameter under starvation conditions (Kim, 1999; Kim and Fogler, 2000). It is also known to produce copious amounts of the exopolymer, dextran, the production of which can be switched on and off by controlling nutrient composition (Jack et al., 1983; Lappan and Fogler, 1992). That is, when monosaccharides are used as the carbon source, cell growth is promoted without exopolymer production. However, when sucrose is used, water-insoluble dextran is produced. 
The second factor for successful application of BPM is to ensure that adequate amounts of nutrient reach the entire region so that cell growth and exopolymer production are sufficient to create a plug throughout the watered-out zone. Previous research has demonstrated the feasibility of nutrient transport (Jack and Stehmeier, 1988; Jenneman et al., 1984). Finally, the established plug must remain stable during starvation induced by water flooding; Kim and Fogler (2000) have demonstrated this stability.

L. mesenteroides is well suited for biomass plugging studies because of the growth characteristics just described. To apply and understand these characteristics in field-scale reservoirs, the physics of pore-scale propagation need to be understood. To achieve this understanding, micromodels were used in this research. The plugging capabilities of the biomass it produces have been demonstrated in parallel core flood and parallel micromodel experiments (Jack and DiBlasio, 1985; Kim, 1999, Lappan and Fogler, 1992). The kinetics of growth and dextran production using sucrose nutrient have been determined (Lappan and Fogler, 1994). Furthermore, the rate at which the permeability is reduced as the biomass plug develops has been predicted using a continuum model (Lappan and Fogler, 1996) and a network model (Kim and Fogler, 2000). Adverse nutrient conditions that inhibit the growth and the development of a biomass plug have been investigated (Wolf and Fogler, 2001). Starvation under other adverse conditions - such as extreme $\mathrm{pH}$, concentration of trace minerals, and temperature-have also been determined (Kim, 1999; Kim and Fogler, 2000).

Although much is known about L. mesenteroides and use of it to reduce permeability in porous media, this body of research identified additional technical issues. During core flood experiments by Lappan and Fogler (1992), oscillations in the pressure drop across the porous media occurred at constant nutrient injection rates. The physical phenomena associated with these oscillations showed that the oscillations helped propagate biomass more deeply into the porous media (Stewart and Fogler, 2001). A conceptual model (Fig. 1), based on results from packed bed and micromodel experiments, has been developed that describes the mechanisms for plug development and propagation in porous media. It was observed that changes in pressure drop resulting from biomass plugging occur in three phases: the exopolymer-induction phase; the plugging phase; and the plug-propagation phase. The key characteristic associated with the induction phase was a small, but constant, pressure drop across the porous media. During this phase, cell growth and exopolymer (in this case, dextran) production occurred. In the micromodel experiments, colonies consisting of bacteria and dextran were observed. These colonies were scattered throughout the pore bodies and pore throats comprising the porous media. Although the colonies increased in size

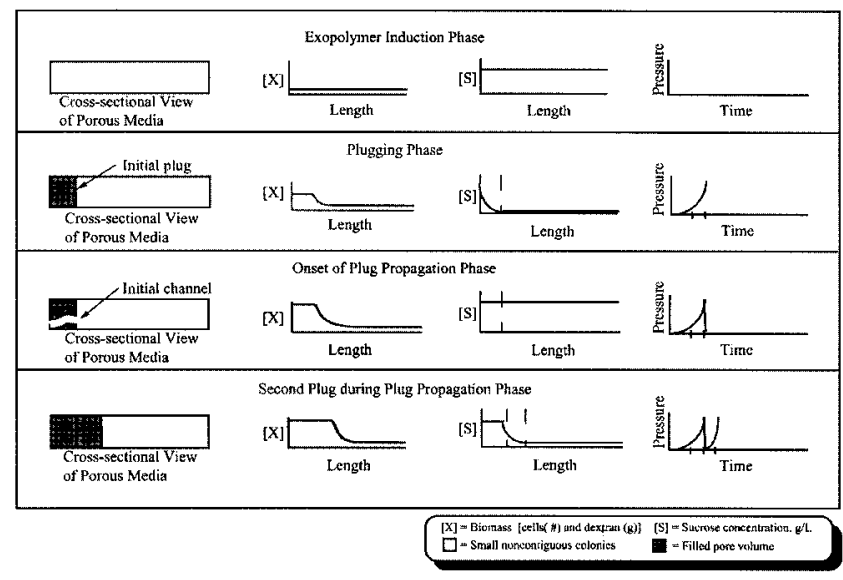

Figure 1. Summary description of biomass plug development and propagation in porous media.

during the exopolymer-induction phase, they were not contiguous and did not fill the entire pore volume of the porous media. In the plugging phase, the key characteristic was an increase in pressure drop across the porous media to some maximum value. Physically the pore throats near the nutrient-inoculum interface were filled with biomass as the colonies increased in size until they became contiguous, resulting in flow constriction and an increase in pressure drop. In the plug-propagation phase, the pressure drop increased to a point where the plug at the nutrient-inoculum interface began to propagate through the porous media in the direction of flow. The key characteristic of the plug-propagation phase was a series of oscillations in pressure drop across the porous media as nutrient injection continued at a constant flowrate. In micromodel experiments, it could be seen that these oscillations were associated with sequential development of biomass plugs in the direction of flow and channel breakthroughs within each of these plugs (Fig. 1). Specifically, the maximum pressure drop reached during a pressure oscillation was associated with development of a plugged region within the porous media. The minimum pressure drop reached during a pressure oscillation indicated development of a breakthrough channel (or percolation path). The biomass behaved in a manner similar to a Bingham plastic, yielding at a critical shear stress through a series of pore throats and pore bodies to create a breakthrough channel. This study - based on a series of micromodel experiments - addresses the pore-scale physics controlling biomass growth and the formation of a breakthrough channel that leads to plug propagation.

\section{MATERIALS AND METHODS}

\section{Bacteria}

The bacterium used in these experiments is L. mesenteroides (NRRL B523; ATCC 14935). It is a facultative 
anaerobe that grows under mesophilic conditions. The cells are coccoidal with a diameter of $1 \mu \mathrm{m}$. Cells are present as singlets, doublets, and short chains under high nutrient conditions. The species produces dextran, a water-insoluble exopolysaccharide, when sucrose is used as the primary carbon source. When a stoichiometrically equivalent nutrient containing glucose and fructose is used, the cells undergo growth at the same rate without dextran production (Lappan and Fogler, 1994). The cells for the inoculum were prepared from a stock culture stored at $-40^{\circ} \mathrm{C}$. The inoculum was grown in a batch reactor with nutrient containing $7.9 \mathrm{~g} / \mathrm{L}$ each glucose and fructose, salts, trace minerals, and $10 \mathrm{~g} / \mathrm{L}$ yeast extract. Full details on composition of the nutrient solution have been provided by Lappan (1994).

\section{Equipment}

Micromodels, which are two-dimensional flow networks that simulate porous media, were used in these experiments. A triangular flow network with a coordination number of six was etched in glass using the method described by Wan et al. (1994). A clean network is shown in Figure 2. Pore throats ranged from 55 to $150 \mu \mathrm{m}$ in diameter in a heterogeneous mix with lengths of $300 \mu \mathrm{m}$. The pore bodies were $300 \mu \mathrm{m}$ in diameter. The pore volume was typically 0.35 to $0.4 \mathrm{~mL}$, varying slightly from micromodel to micromodel. The flow region was $3.1 \mathrm{~cm}$ in width and $16.2 \mathrm{~cm}$ in length corresponding to a network of pore bodies that was 40 pore bodies in width and 200 pore bodies in length. The micromodel system shown in Figure 2 was assembled,

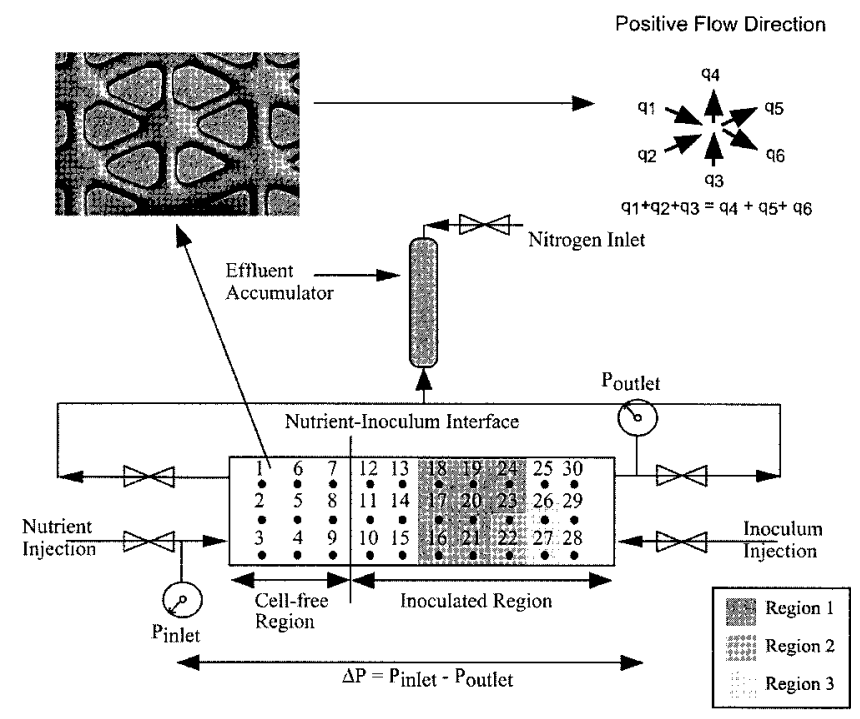

Figure 2. The micromodel system showing the 30 position indicators that allowed mapping of biomass distribution with time from the video recordings. The areas marked Regions 1,2 , and 3 correspond to the area of plugging during development of the first three plugs. Positive nutrient flow at each pore body is shown resulting in a net flow from left to right at the macroscopic scale. sterilized in an autoclave, and placed under a stereomicroscope with a camera connected to a video recorder that took manual and automatic recordings. Pressure transducers that were connected to a data recorder were placed at the inlet and outlet. Positive flow was from left to right at the macroscopic scale. On the pore scale, each pore body was an intersection for six pore throats with positive flow in the direction shown in Figure 2.

\section{Procedure}

A series of micromodel experiments was conducted using L. mesenteroides. The micromodel was saturated by flowing the saturation solution from the inlet to outlet as a back pressure of $100 \mathrm{kPa}$ was applied. The saturation solution contained buffers and yeast extract per Lappan (1994). The concentrated inoculum was then hand injected through the back port shown in Figure 2 until it reached the midway point marked as the nutrient-inoculum interface. Contamination of the inlet nutrient line and inlet face of the micromodel with the inoculum was avoided using this approach and resulted in biomass growth only in the downstream half of the micromodel. The system was shut (i.e., no flow) in for $30 \mathrm{~min}$, and then the saturation solution was injected through the inlet line at $0.015 \mathrm{~mL} / \mathrm{min}$ for an additional $30 \mathrm{~min}$ to sweep planktonic cells from the micromodel network. At $\mathrm{t}=0$, nutrient solution containing sucrose as the carbon source was injected at a constant flowrate through the nutrient inlet lines (Fig. 2) and continued throughout the experiment.

Pressure was monitored at 15-min intervals using a computer-controlled system and recorder. No effluent samples were collected from this closed system. During plug development, video recordings at $100 \times$ magnification were taken at the 30 position indicators shown in Figure 2 at selected timepoints throughout the experiment. In addition, full visual sweeps of the micromodel at magnifications of $10 \times$ were recorded on video at timepoints of interest. The 30 position indicators were used to locate the biomass distributed across the micromodel and translate this information into percolation maps for visual analysis of biomass distribution during plug and channel formation. The maps were simplified by focusing on the location of the first three breakthrough channels.

\section{Experimental Conditions}

Three different flowrates at a reference sucrose concentration of $15 \mathrm{~g} / \mathrm{L}$ and three different sucrose concentrations at a reference flowrate of $0.015 \mathrm{~mL} / \mathrm{min}$ were used. The experimental conditions are summarized in Table I. Experiment ME-17b is the base case. 
Table I. Sucrose concentrations and flowrates used in the micromodel experiments.

\begin{tabular}{cccc}
\hline Experiment number & Sucrose concentration $(\mathrm{g} / \mathrm{L})$ & Volumetric flowrate $(\mathrm{mL} / \mathrm{min})$ & Comments \\
\hline ME-17b & 15 & 0.015 & $\begin{array}{l}\text { Base-case conditions; video-tape } \\
\text { footage used to document succes- } \\
\text { sive plug and channel formation }\end{array}$ \\
ME-17d & 15 & & Flowrate varied \\
ME-19 & 15 & 0.05 & Flowrate varied \\
ME-18a & 5 & 0.005 & Sucrose conc. varied \\
ME-18f & 30 & 0.015 & Sucrose conc. varied \\
ME-18g & 1 & 0.015 & Sucrose conc. varied \\
\hline
\end{tabular}

\section{RESULTS AND DISCUSSION}

This research used micromodel experiments to understand the pore-scale physics of biomass plug development and propagation in porous media under nutrient-rich conditions. The pressure oscillations in the micromodel experiments mimic those observed previously in porous sandstone core-flood and packed-bed experiments (Lappan and Fogler, 1992; Stewart and Fogler, 2001). The micromodel experiments determined: (1) the characteristics of biomass distribution that caused plugging; (2) the reason for the location of a breakthrough channel along a specific path, including the role of pore bodies in channel formation; and (3) the yield stress required to cause biofilm to shear and create a breakthrough channel.

\section{Observations}

The pressure drop across the micromodel is shown as a function of time and pore volumes injected for the base case under constant flowrate conditions in Figure 3. The characteristic exopolymer-induction phase, plugging phase, and plug-propagation phase are readily observed in Figure 3. The location, or regions, of the first three plugs associated with the first three maximum pressure

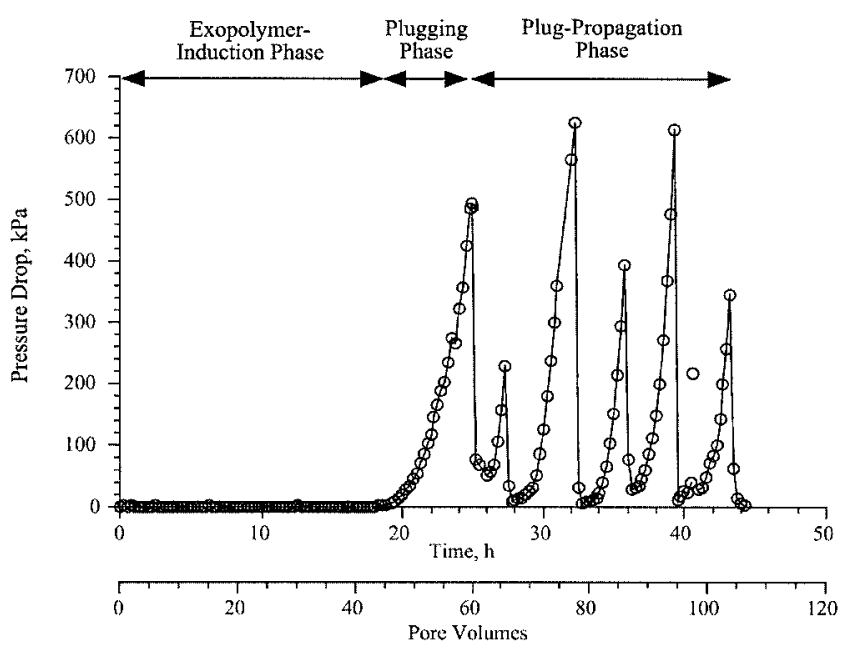

Figure 3. Pressure drop across the micromodel as a function of time and pore volumes of sucrose injection at a sucrose concentration of $15 \mathrm{~g} / \mathrm{L}$ and a flowrate of $0.015 \mathrm{~mL} / \mathrm{min}$. drop values are identified in Figure 2. Region 1 corresponds to the area of the first plug that encompassed 4120 pore throats. Region 2 corresponds to the area of the second plug that developed after breakthrough of the first plug. The second plug encompassed an additional 1027 pore throats. Similarly, Region 3 corresponds to the area of the third plug that encompassed an additional 1888 pore throats. The plug development in the micromodel is consistent with the conceptual model for sequential plug development in the direction of flow propagated by periodic formation of breakthrough channels described in Figure 1.

During plug development, isolated groupings of empty pore throats, which often contained empty pore bodies, were observed. The topography of the developing plug was characterized by determining the fraction of empty pore throats, $f_{i p}$. During development of the breakthrough channel, consecutive, empty pore throats formed a percolating backbone with nonconducting branches. The topography of a breakthrough channel was characterized by determining the fraction of empty pore throats in the breakthrough channel, $f_{N}$, and in the percolating backbone alone, $f_{N b}$. Comparison of $f_{N}$ and $f_{N b}$ indicates the degree of branching of the breakthrough channel. When $f_{N}=f_{N b}$, branching does not occur.

\section{Characteristics of Biomass Distribution That Caused Plugging}

The distribution of biomass during development of the first three plugs and breakthrough channels are presented as percolation maps of the micromodel in Figures 4 through 9. The video record of biomass distribution was translated graphically to create these percolation maps. The biomass distribution for the first plug that occurred after $23.5 \mathrm{~h}$ of sucrose injection is shown in Figure 4 for a typical area within Region 1. This plug corresponds to the first rise in pressure shown in Figure 3 just before breakthrough occurs. In this figure, the triangular network of the micromodel is depicted with the untextured lines (e.g., /and $\backslash$ ), representing pore throats that are either full or partially full of biomass. A pore throat was considered partially full when any amount of biomass was visible in it. Typically, this biomass bridged the pore throat but did not extend 


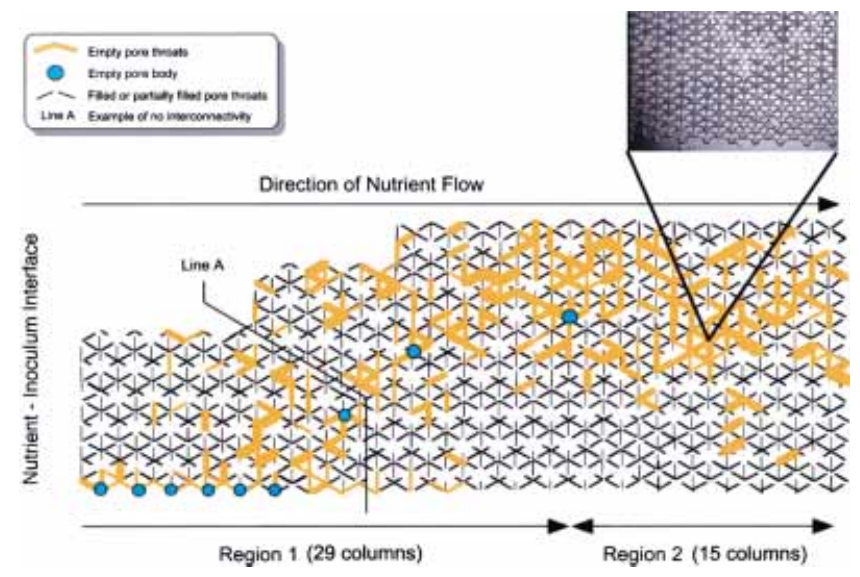

Figure 4. Biomass distribution during development of the first plug after $23.5 \mathrm{~h}$ of sucrose injection. Line $A$ is an example of where there is no connectivity between empty pore throats. [Color figure can be viewed in the online issue, which is available at www.interscience. wiley.com.]

the full length of the pore throat. The textured lines and circles (e.g., $\boldsymbol{\wedge}$ and $\bullet$ ) represent pore throats and pore bodies, respectively, that are still empty. At $23.5 \mathrm{~h}$, these empty pore spaces are isolated groupings that do not form a conductive channel across the plug. Line $A$ in Figure 4 represents an example of an area where there is no interconnectivity between empty pore throats. Figure 5 shows the channel of connected, empty pore throats in Region 1 immediately after breakthrough of the first plug. Figure 6 shows the development of the second plug. Here the biomass refilling of the breakthrough channel in the first plug as well as the filling of more pore throats in Region 2 were observed. Biomass filling in both these regions results in the formation of the second plug and corresponds to the second rise in pressure shown in Figure 3. The breakthrough of the second plug is shown in Figure 7. Figures 8 and 9 show similar trends for the formation and breakthrough of the third plug. These percolation maps for the second

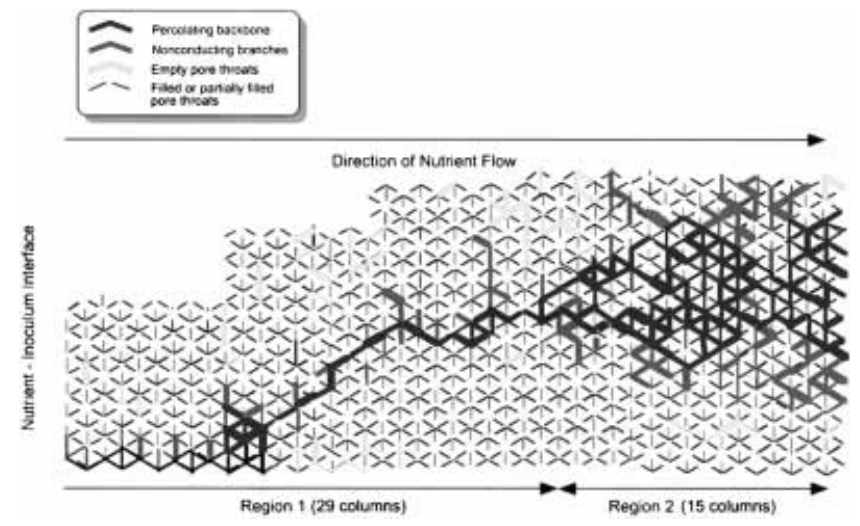

Figure 5. Biomass distribution after formation of the first breakthrough channel after $26 \mathrm{~h}$ of sucrose injection. [Color figure can be viewed in the online issue, which is available at www.interscience. wiley.com.]

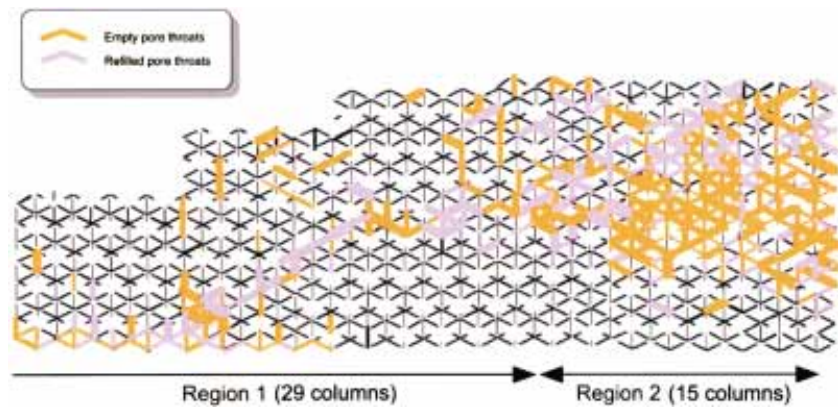

Figure 6. Biomass distribution during development of the second plug that formed due to biomass refilling of the breakthrough channel in Region 1 and biomass filling of empty pores in Region 2 between 26 and $27.75 \mathrm{~h}$ of sucrose injection. [Color figure can be viewed in the online issue, which is available at www.interscience.wiley.com.]

and third plugs correspond to position indicators 21 and 22, and position indicators 21, 22, and 27 (Fig. 2), respectively. All three percolation maps were used to determine the topography and make conclusions about the pore-scale physics of biomass plug propagation.

The topography of a biomass plug is described by the fraction of empty pore throats, $f_{i p}$. The $f_{i p}$ was determined and is presented in Table II for the first three biomass plugs. For example, the first row and first two columns show the fraction of empty pore throats in Region 1 and Plug 1 just before channel breakthrough. The next three columns of the first row show the fraction of empty pore throats in Regions 1 and 2 that comprise Plug 2 just before the second channel breaks through.

As was previously described, the micromodel network had a distribution of pore throat diameters. There were six pore throat sizes: $56.3 \mu \mathrm{m}, 75 \mu \mathrm{m}, 93.8 \mu \mathrm{m}, 113 \mu \mathrm{m}$, and $150 \mu \mathrm{m}$. There were three exit pore throats from each pore body that had some combination of these six diameters. As each pore body and set of exit pore throats were examined, the diameters were lumped into three size classifications: small, medium, and large. The size of the empty pore throats was classified using this scheme, and the results are expressed in Table II.

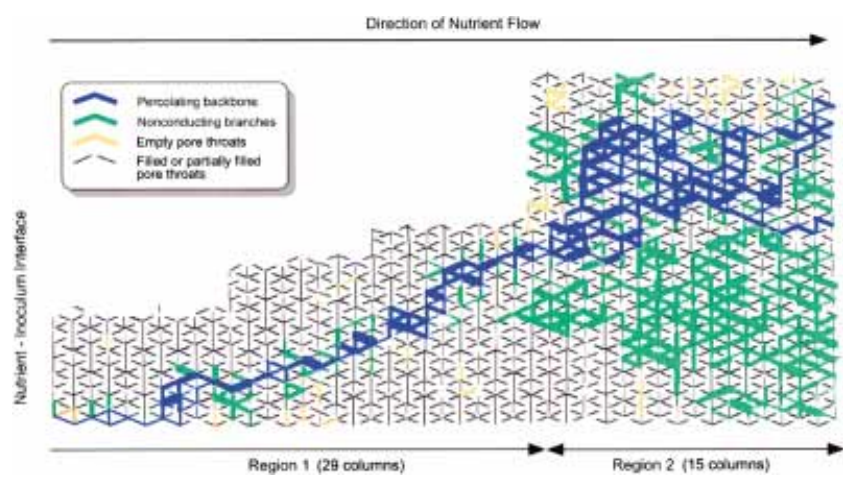

Figure 7. Biomass distribution after formation of the second breakthrough channel at $27.75 \mathrm{~h}$ of sucrose injection. [Color figure can be viewed in the online issue, which is available at www.interscience. wiley.com.] 


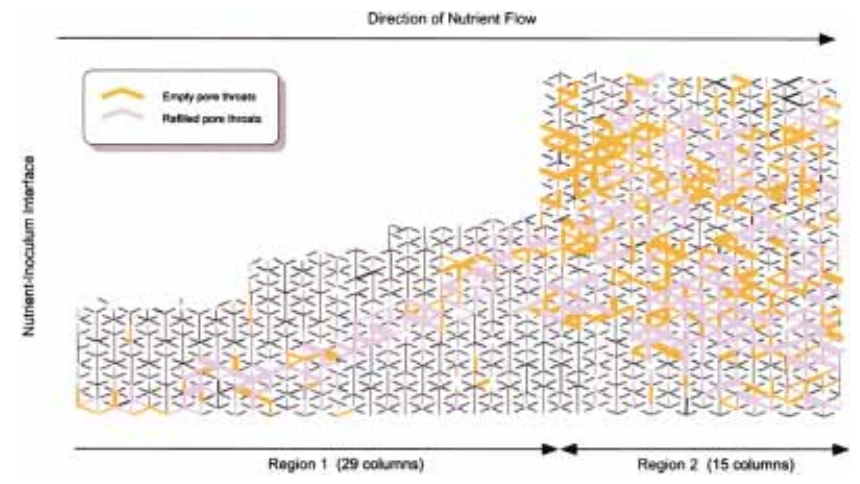

Figure 8. Biomass distribution in Regions 1 and 2 during development of the third plug due to biomass refilling of the breakthrough channel between 27.75 and $32.5 \mathrm{~h}$ (Region 3 not shown). [Color figure can be viewed in the online issue, which is available at www.interscience. wiley.com.]

For example, row 2 and column 4 shows that $55 \%$ of the small pore throats were empty in Region 2 in Plug 2 just before breakthrough. Four observations can be made from these results:

- The distribution of biomass during the development of the first plug.

- The relationship of empty pore throats downstream of a developing plug to the location of breakthrough channel.

- The distribution of biomass during development of the second and the third plugs.

- A comparison of the fraction of empty pore throats in each region as the plug propagated.

\section{Distribution of Biomass During Development of First Biomass Plug}

It was observed that during development of the initial plug, the largest fraction of empty pore throats, $f_{i p \text {, }}$ contained those with the smallest pore throat radii. This result indicates that the larger diameter pore throats filled first with biomass followed by the smaller pore throats as the first plug developed. A similar trend was also observed for the second and third plugs (Table II).

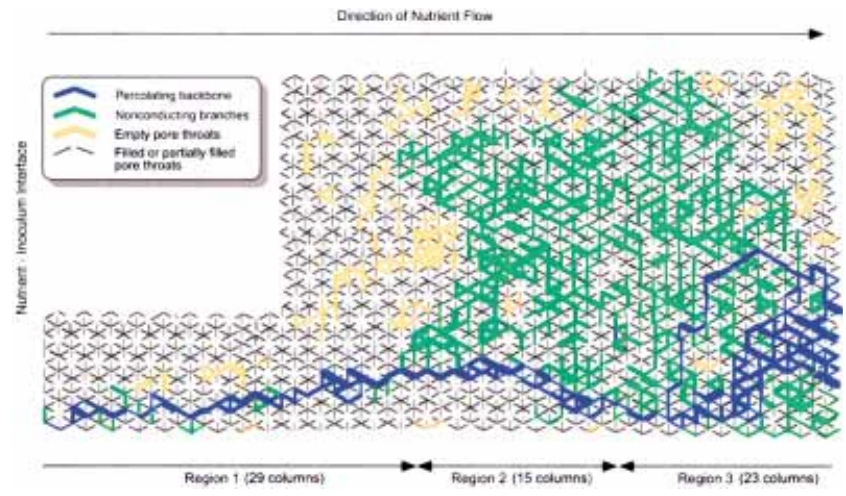

Figure 9. Biomass distribution after formation of the third breakthrough channel after $32.5 \mathrm{~h}$. [Color figure can be viewed in the online issue, which is available at www.interscience.wiley.com.]

For a constant volumetric flowrate, the substrate loading and, hence, the rate of growth is greatest in the larger pore throats. This result in supported by previous coreflood experiments (Lappan and Fogler, 1992; Torbati et al., 1986). Because of the distribution of pore sizes, there are dominant flow paths through the micromodel. As nutrient injection and biomass growth proceeded, these dominant flow paths, which initially consisted of consecutive large pore throats, became filled with biomass. Consequently, flow was diverted to the smaller consecutive pore throats resulting in a shift in the location of the dominant flow path. Therefore, as the plug developed and large pores were closed, fewer pathways became available. Consequently, the bulk of the nutrient flowed through the smaller pore throats, causing the average pore throat velocity and local shear stresses to increase in these pore throats.

\section{Relationship of Empty Pore Throats Downstream of a Developing Plug to Location of Breakthrough Channel}

The increased local shear stresses in the smaller pore throats just described led to a second observation: The location of the subsequent breakthrough channel is related to the location of empty pore throats at the back

Table II. Distribution of biomass during plug development just before channel breakthrough.

\begin{tabular}{|c|c|c|c|c|c|c|c|c|c|c|}
\hline & & $\begin{array}{c}(1) \\
\text { Region } 1\end{array}$ & $\begin{array}{c}(2) \\
\text { Plug } 1^{\mathrm{a}}\end{array}$ & $\begin{array}{c}(3) \\
\text { Region 1 }\end{array}$ & $\begin{array}{c}(4) \\
\text { Region } 2\end{array}$ & $\begin{array}{l}\text { (5) } \\
\text { Plug } 2^{\mathrm{a}}\end{array}$ & $\begin{array}{c}(6) \\
\text { Region 1 }\end{array}$ & $\begin{array}{c}(7) \\
\text { Region } 2\end{array}$ & $\begin{array}{c}(8) \\
\text { Region } 3\end{array}$ & $\begin{array}{c}\text { (9) } \\
\text { Plug } 3^{\mathrm{a}}\end{array}$ \\
\hline (1) & $f_{i p}{ }^{\mathrm{c}}$ & 0.028 & 0.028 & 0.019 & 0.157 & 0.047 & 0.018 & 0.255 & $\mathrm{NA}^{\mathrm{b}}$ & $0.066^{\mathrm{b}}$ \\
\hline (2) & $\begin{array}{l}\text { Smallest } r \\
(\%)^{\mathrm{d}}\end{array}$ & 63 & 63 & 62 & 55 & 57 & 68 & 64 & $\mathrm{NA}^{\mathrm{b}}$ & $65^{\mathrm{b}}$ \\
\hline (3) & $\begin{array}{l}\text { Medium } r \\
(\%)^{\mathrm{d}}\end{array}$ & 10 & 10 & 20 & 13 & 15 & 12 & 11 & $\mathrm{NA}^{\mathrm{b}}$ & $14^{\mathrm{b}}$ \\
\hline (4) & $\begin{array}{l}\text { Largest } r \\
(\%)^{\mathrm{d}}\end{array}$ & 27 & 27 & 18 & 32 & 28 & 20 & 25 & $\mathrm{NA}^{\mathrm{b}}$ & $31^{\mathrm{b}}$ \\
\hline
\end{tabular}

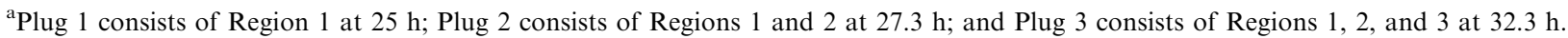

${ }^{\mathrm{b}}$ Data not available for Region 3 of Plug 3; reported data are only from Regions 1 and 2 in Plug 3.

${ }^{\mathrm{c}} f_{i p}$ is the fraction of empty pore throats within a region of the micromodel plugged with biomass.

$\mathrm{d}_{r}=$ pore throat radius.
} 
boundary of the developing plug. Video recordings revealed that just before and during formation of the breakthrough channel, the biomass in an area downstream of the developing plug always experienced localized shearing. For example, in Figure 4, biomass distribution is presented for both Regions 1 and 2. The plug was located in Region 1. Nevertheless, biomass growth was also occurring in Region 2, albeit at a slower rate due to lower sucrose concentrations resulting from its consumption in Region 1 (see Fig. 1). This activity of growth and shearing at the back boundary of the plug along with the location and number of empty pore throats upstream gives rise to the location of the next breakthrough channel. The reason for localized shearing at the back boundary of the plug is discussed here; the relationship between the location of empty pore throats in the upstream plug and the breakthrough channel is discussed in the next section.

Just before breakthrough, pore throats at the back boundary of Region 1 are being emptied (Fig. 4) due to local shearing. This biomass shearing activity is believed to be related to the level of sucrose concentration in Region 2. To confirm this hypothesis, a series of experiments were carried out in which the inlet sucrose concentration was varied while the volumetric flowrate was held constant (Table I). Four different inlet sucrose concentrations were used, and the pressure drop across the micromodel was recorded and is shown in Figure 10. For an inlet concentration of $1 \mathrm{~g} / \mathrm{L}$ no plug developed and no maximum pressure drop was observed. As can be seen in Figure 10, the value of the maximum pressure drop indicated by the circles increased with inlet sucrose concentration and, hence, total sucrose injected.

These results are consistent with the findings of Wolf and Fogler (2001) who determined that the amount of high-molecular-weight (i.e., insoluble) dextran increased with increasing sucrose concentrations. This higher molecular weight and resulting decreased solubility in-

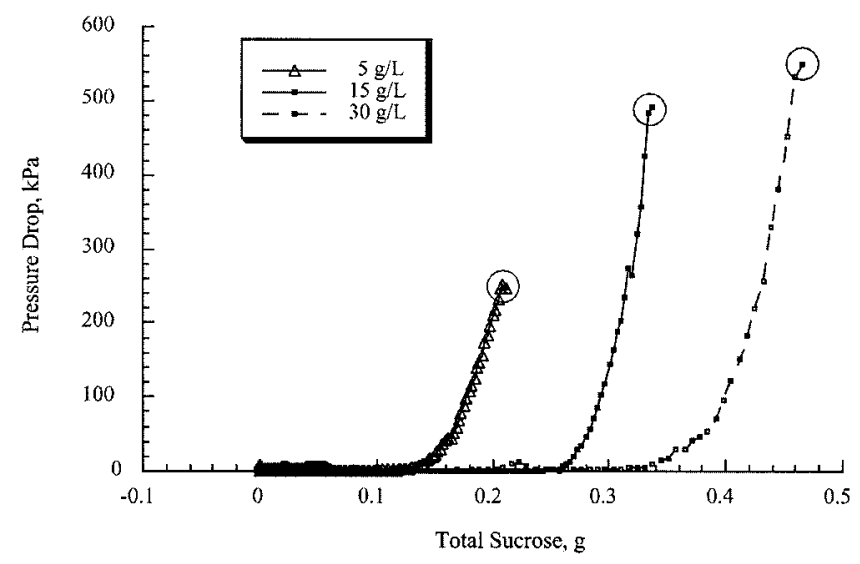

Figure 10. Maximum pressure drop at first channel breakthrough (circles) under constant flowrate conditions as a function of inlet sucrose concentration. dicate a more highly branched dextran that is more resistant to shear stresses. Consequently, as the inlet sucrose concentration is increased, a higher maximum pressure drop just before channel breakthrough would be expected. In addition, Kim and Fogler (2000) observed that a critical shear stress existed and increased with increasing total sucrose injected.

These experimental results along with the observations of Kim and Fogler (2000) and Wolf and Fogler (2001) suggest that the localized shearing activity of biomass in regions downstream of the developing plug just before breakthrough was due to lower sucrose concentrations. Specifically, biomass formed in Region 2 during development of the first plug and in Region 3 during development of the second plug had lower molecular weight dextran with less branching, making the biomass weaker and more susceptible to shearing. Moreover, during the formation of the first plug, the average pore throat velocity was increasing as a dominant flow path directed the flow of nutrients through fewer pathways made up of smaller pore throats in the upstream plug and caused flow to funnel into Region 2. Because the flow entering Region 2 was being funneled to the regions of weaker biomass, biomass was emptied from pore throats in the opposite direction to the nutrient flow until the front of the plug was reached and the breakthrough channel formed.

\section{Distribution of Biomass During Development of Second and Third Biomass Plugs}

A third observation was that, after breakthrough and during development of the second and third plugs, biomass filled the breakthrough channel as well as the empty pore throats in the region just downstream of the previous plug's boundary. Comparing Figure 5 with Figure 6, we see the refilling of the first plug breakthrough in both the percolating backbone and the nonconducting branches. Similarly, refilling of the second plug breakthrough can be seen by comparing Figures 7 and 8 . Specifically, $50 \%$ of the percolating backbone and $35 \%$ of the nonconducting branches in the first breakthrough channel were refilled with biomass. Likewise, during development of the third plug, $43 \%$ of the percolating backbone and $50 \%$ of the nonconducting branches in the second breakthrough channel were refilled.

Filling of nonconducting branches indicates that nutrient and detached bacteria reached the empty pore throats either by localized backflow or percolation through near-filled pore throats. Both of these phenomena are possible. Video recordings revealed brief periods of negative flow in some pore throats as plugging proceeded. It is plausible to assume that negative flow occurred when a pore throat became plugged, causing a momentary backflow. In addition, studies using confocal laser microscopy have shown that a 
primitive circulatory system exists within biofilms that allow convective flow through water channels formed at the base of biofilm structures (Costerton, 1999; Costerton et al., 1994). It is plausible to assume nutrient accessed nonconducting branches through the primitive circulatory system; that is, convective flow moved nutrient through the biomass filled pores through this circulatory system and into adjacent empty pore throats comprising nonconducting branches.

\section{Comparison of Fraction of Empty Pore Throats in Each Region as Biomass Plug Propagated}

The fourth observation is that the degree of plugging (i.e., maximum pressure drop reached before channel breakthrough) is related to the amount and distribution of biomass. As can be seen in Figure 3, the second maximum pressure drop was less than the first maximum pressure drop reached before channel breakthrough. To determine the reason for this difference, comparison of the fraction of empty pore throats, $f_{i p}$, for Region 2 was compared to Region 1. Region 1 changed very little with respect to quantity or distribution of biomass between the first and second plugs (Table II). Region 1 always had the smallest number of empty pore throats (i.e., lowest $f_{i p}$ ) (Table II). On the other hand, the fraction of empty pore throats, $f_{i p}$, in Region 2 during development of the second plug was an order of magnitude higher than the $f_{i p}$ of Region 1 (Table II). The reason was differences in the initial distribution of biomass. Unlike Region 1, biomass in Region 2 was not uniformly distributed at the onset of the second plug. As previously discussed, the localized shearing at the back boundary of the first plug resulted in biomass filling only a few pore throats. Thus, as biomass growth proceeded, the plug did not develop uniformly; instead, growth occurred predominantly in those pore throats that still had biomass in them. As a result, areas in Region 2 often contained a single or very few consecutive pore throats that were filled with biomass between areas of large, isolated groupings of empty pore throats. This partial plugging was sufficient to cause the onset of a pressure increase; however, owing to the paucity and location of biomass in Region 2, the second channel broke through at a lower pressure drop.

This result suggests that biomass plugs with a high fraction of empty pore throats, $f_{i p}$, can be unstable, allowing channels to breakthrough a plug at lower than expected normal pressures if the biomass distribution is sparse. In this context, sparseness reflects the location of biomass-filled pore throats and the number of consecutively filled pore throats (i.e., the extent of the plugged area). In turn, the location of filled pore throats is a function of pore-scale parameters such as the pore throat diameter, a physical characteristic of the porous media. It can be surmised that the stability of the plug may differ with different pore-scale characteristics of the porous media, which could include not only pore throat diameter, but tortuosity and connectivity.

\section{Location of Breakthrough Channels and Role of Pore Bodies in Channel Formation}

The topography of the first three breakthrough channels described in terms of fraction of empty pore throats in the breakthrough channel, $f_{N}$, and the percolating backbone, $f_{N b}$, are presented and identified in the upper portion of Table III. For example, the first row and first two columns show the fraction of empty pore throats in Region 1 and Plug 1. The next three columns of the first row show the fraction of empty pore throats in Regions 1 and 2 that comprise the second breakthrough channel. Similarly, the fraction of empty pore throats in the percolating backbone of the breakthrough channel is shown in row 2 . As in the analysis of the topography of the plugs, the pore distributions were lumped into three sizes and the size classification of empty pore throats at each pore body was determined. For example, row 3 and column 1 indicate that $84 \%$ of the empty pore throats from the previous plug that had been incorporated into the

Table III. Distribution of biomass after channel breakthrough.

\begin{tabular}{|c|c|c|c|c|c|c|c|c|c|c|}
\hline & & $\begin{array}{c}(1) \\
\text { Region } 1\end{array}$ & $\begin{array}{c}(2) \\
\text { Channel } 1^{\mathrm{a}}\end{array}$ & $\begin{array}{l}(3) \\
\text { Region } 1\end{array}$ & $\begin{array}{c}(4) \\
\text { Region } 2\end{array}$ & $\begin{array}{c}(5) \\
\text { Channel } 2^{\mathrm{a}}\end{array}$ & $\begin{array}{c}(6) \\
\text { Region } 1\end{array}$ & $\begin{array}{c}(7) \\
\text { Region } 2\end{array}$ & $\begin{array}{c}(8) \\
\text { Region } 3\end{array}$ & $\begin{array}{c}(9) \\
\text { Channel } 3^{\mathrm{a}}\end{array}$ \\
\hline (1) & $f_{N}$ & 0.024 & 0.024 & 0.038 & 0.465 & 0.123 & 0.019 & 0.350 & 0.278 & 0.137 \\
\hline (2) & $f_{N b}$ & 0.015 & 0.015 & 0.020 & 0.165 & 0.049 & 0.012 & 0.034 & 0.065 & 0.029 \\
\hline \multicolumn{11}{|c|}{ Empty pore throats from isolated groupings forming breakthrough channel ${ }^{\mathrm{b}}$} \\
\hline (3) & Smallest $r(\%)$ & 84 & 84 & 83 & 49 & 58 & 63 & 67 & 52 & 54 \\
\hline (4) & Medium $r(\%)$ & 16 & 16 & 0 & 22 & 16 & 0 & 8 & 10 & 9 \\
\hline (5) & Largest $r(\%)$ & 0 & 0 & 17 & 28 & 26 & 37 & 25 & 38 & 37 \\
\hline \multicolumn{11}{|c|}{ Pore throats where biomass was sheared to form breakthrough channel ${ }^{\mathrm{c}}$} \\
\hline (6) & Smallest $r(\%)$ & 54 & 54 & 58 & 62 & 60 & 38 & 61 & 80 & 57 \\
\hline (7) & Medium $r(\%)$ & 17 & 17 & 14 & 8 & 10 & 15 & 13 & 10 & 13 \\
\hline (8) & Largest $r(\%)$ & 29 & 29 & 28 & 30 & 30 & 47 & 26 & 10 & 30 \\
\hline
\end{tabular}

${ }^{\text {a}}$ Channel 1 through Plug 1, Channel 2 through Plug 2, and Channel 3 through Plug 3.

${ }^{\mathrm{b}}$ Pore throats empty during previous plug that comprise the percolating backbone.

${ }^{\mathrm{c}}$ Biomass sheared from pore throats as breakthrough channel formed. 
breakthrough channel were classified as small. Similarly, row 6 and column 1 of Table III indicate that $54 \%$ of the pore throats from which biomass was sheared to form a breakthrough channel were classified as small throats.

\section{Characteristics of Breakthrough Channels}

A breakthrough channels forms when a maximum pressure drop value is reached and biomass, which acts in a manner similar to a Bingham plastic, is sheared from a series of consecutive pore throats. The relationship between pressure drop across the porous media and wall shear stress is obtained form a force balance and is:

$$
\tau_{w}=\frac{P_{N} A_{N}}{A_{S}}=\frac{P_{N} R}{2 L}
$$

where $\tau_{\mathrm{w}}$ is wall shear stress, $P_{N}$ is the normal pressure, $A_{\mathrm{N}}$ is the area normal to the imposed pressure, $A_{S}$ is the surface area of the pore throat wall on which the yield stress is acting, $R$ is the pore throat radius, and $L$ is the length of the pore throat, which is constant for these experiments. The stress at any radius, $r$, is:

$$
\tau_{w}=\frac{P_{N} r}{2 L}
$$

If we assume that the Bingham yield stress, $\tau_{B}$ is equal to $\tau_{\mathrm{w}}$ when biomass yields to form a breakthrough channel, we can estimate the $\tau_{B}$ for the biomass. Using this assumption an interesting observation can be made. As can be seen in Table III, the highest fraction of pore throat radii from which biomass was sheared to form the breakthrough channel are the smallest diameter pore throats. The wall shear stress is the smallest for the smallest diameter pore throats as can be seen in Eq. (1). If the channel is forming along the smaller diameter pore throats, it can be assumed that the weakest biomass is located in these pore throats. This assumption is plausible because there is a lower substrate loading in the smaller diameter pore throats, resulting in lower total sucrose being provided to the biomass. In turn, a weaker dextran is produced, resulting in lower yield stress, as seen in Figure 10. Hence, it is reasonable that the breakthrough channels form along a path of pore throats with the smallest radii. Implicit in these statements is that the pore throats are completely full of biomass and that all biomass is removed from a pore throat at the time of yielding.

Biomass was sheared from larger pore throats only when one of four situations arose:

- An empty pore throat or a group of empty pore throats that were incorporated into a breakthrough channel were separated by a single, large pore throat filled with biomass. In this case, shearing the biomass from this large pore throat created the percolating path. An example of this situation can be seen by comparing Figure 4 with Figure 5 .
- An empty pore throat or a group of empty pore throats that were incorporated into a breakthrough channel were connected by a pathway that had the smallest wall surface area when compared with alternative potential pathways. An example of this situation can be seen by comparing Figure 4 with Figure 5.

- Biomass from several pore throats at an intersecting pore body yielded simultaneously causing some larger pore throats that had biomass to be sheared. An example of this situation can be seen by comparing Figure 4 with Figure 5.

- Random event.

Similar results were observed for the second and third breakthrough channels shown in Figures 7 and 9, respectively.

These results suggest that breakthrough channels formed along paths of consecutive pore throats with the lowest total surface area of the pore throat walls in contact with biomass. This conclusion is easily confirmed by comparing the total pore throat wall surface areas for the series of connecting pore throats in the actual percolating backbones with possible alternative locations. These alternative locations included the percolating backbone in Region 1 for the second and third channels as well as for a hypothetical percolating backbone translated either one pore throat up or down of the actual percolating backbone. The wall surface areas for actual and these alternative pathways are presented in Table IV for comparison. In cases where the actual and alternative paths had equal surface areas, the breakthrough channel formed along the path that incorporated the most empty pore bodies.

Other characteristics of the breakthrough channels can be observed by comparing fractions of empty pore throats presented in Tables II and III.

- The second and third breakthrough channels were progressively more highly branched than the first breakthrough channel.

- A higher fraction of empty pore throats in a plug corresponds to a higher fraction of empty pore throats in the next breakthrough channel and, consequently, more branching.

- The fraction of empty pore throats in the percolating backbone within Region 2 was decreasing with each successive plug development/channel breakthrough cycle.

These observations indicate that the topography of the breakthrough channel is related to the topography of the plug. Factors, such as pore throat radii and sucrose gradients, that affect distribution of the biomass in a plug set the stage for the location of the breakthrough channel.

\section{Channel Location}

Another observation that can be made about pathways of the breakthrough channels is that they shift with each 


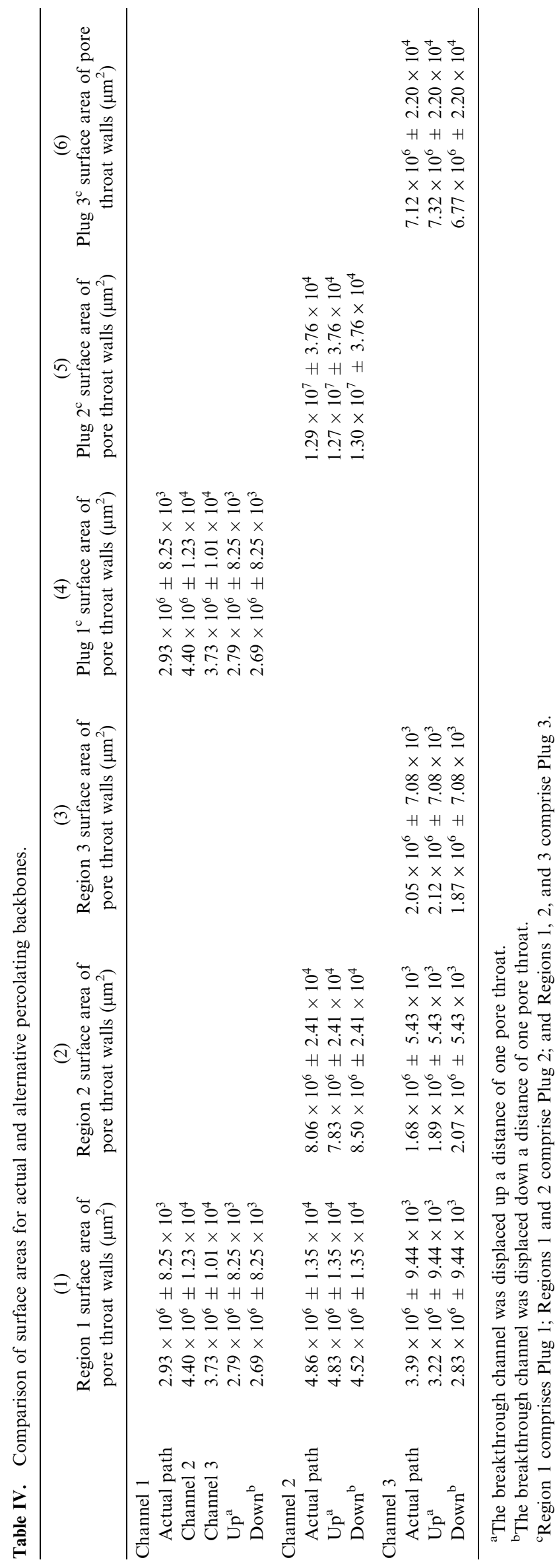

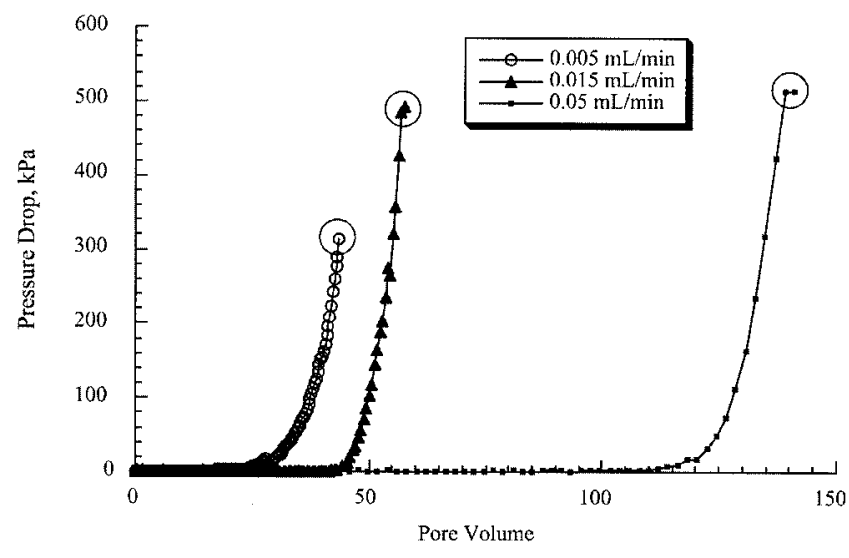

Figure 11. Maximum pressure drop at first channel breakthrough (circles) under constant inlet sucrose concentrations as a function of flowrate.

cycle. The location of the second breakthrough channel shown in Figure 7 includes the same entry pore throats used by the first breakthrough channel shown in Figure 5. However, the second breakthrough channel shifts upward from the location of the first channel in the remainder of Region 1. Likewise, the location of the third breakthrough channel in Regions 1 and 2 has shifted significantly from the location of the second channel as can be seen by comparing Figure 7 with Figure 9. The reason for the shift in location of the breakthrough channels was further explored by evaluating the effects of flowrate. A series of micromodel experiments in which flowrate was varied (see Table I) was conducted. As shown in Figure 11, at the highest flowrate of $0.05 \mathrm{~mL} / \mathrm{min}$ the maximum pressure drop values just before channel breakthrough were higher than that for $0.005 \mathrm{~mL} / \mathrm{min}$, indicating a higher Bingham yield stress for biomass grown at higher average pore throat velocities. As the biomass begins to grow and refill the breakthrough channel, it is exposed to a higher shear stress because the nutrient flow is primarily along the breakthrough channel. It is plausible that biomass grown under higher shear stress will have a higher Bingham yield stress than biomass in adjacent pores, causing the next breakthrough channel to shift to locations of weaker biomass. This result is consistent with other studies on channel flow where biomass was grown and sheared from the surfaces to which it was attached (Stoodley et al., 1999). Namely at flowrates equal to or lower than those at which the biomass was grown, the biomass remained attached to the channel surfaces. If the flowrates were higher than that at which the biomass was grown, the biomass was sheared.

\section{Bingham Yield Stress and Biofilm Shearing}

The Bingham yield stress was calculated for the three biomass plugs and breakthrough channels. Using a force 
balance equation for flow through circular pore throats, the shear stress can be expressed as:

$$
\tau=\frac{8 \eta \bar{U}_{p}}{d}
$$

where $\tau$ is the shear stress, $\eta$ is the viscosity, $\bar{U}_{p}$ is the average pore velocity, and $d$ is the pore throat diameter. Furthermore, assuming Hagen-Poiseuille flow through a tube, $\bar{U}_{p}$ and $d$ can be determined by changes in permeability and pressure drop according to Eqs. (4) and (5):

$$
\begin{gathered}
\bar{U}_{p}=U_{p o}\left(\frac{K}{K_{o}}\right)^{-1 / 2}=U_{p o}\left(\frac{\Delta P_{o}}{\Delta P}\right)^{-1 / 2} \\
d=d_{o}\left|\left(\frac{K}{K_{o}}\right)\right|^{1 / 4}=d_{o}\left(\frac{\Delta P_{o}}{\Delta P}\right)^{1 / 4}
\end{gathered}
$$

$U_{p o}$ is the initial average pore throat velocity, $K / K_{o}$ is the permeability reduction ratio that is inversely proportional (in accordance with Darcy's law) to $\Delta P / \Delta P_{o}$ (the ratio of pressure changes), and $d_{o}$ is the initial average pore throat diameter. The Bingham yield stress was assumed to be equal to the shear stress at the maximum pressure drop value. Using these relationships, the Bingham yield stress in each pore throat was calculated as $2.9 \mathrm{~Pa}$ when $K / K_{\mathrm{o}}$ was 0.0017 as the first channel formed, $1.7 \mathrm{~Pa}$ when $K / K_{o}$ was 0.0035 as the second channel formed, and $3.5 \mathrm{~Pa}$ when $K / K_{o}$ was 0.0013 as the third channel formed. These values compare well with critical shear stress reported for other bacterial species (Powell and Slater, 1982).

\section{CONCLUSIONS}

Micromodels were used to study biomass plug formation and propagation in porous media. The trends observed in micromodel experiments were identical to those observed in porous core-flood and packed-bed experiments (Lappan, 1994; Lappan and Fogler, 1992; Stewart and Fogler, 2001). A series of micromodel experiments on the pore-scale physics indicated that a biomass plug can be propagated through porous media with continued nutrient injection to depths beyond the nutrient-inoculum interface. These greater depths of plug development are needed for BPM applications. This propagation is possible because: (1) biomass is continuously generated by in situ growth; and (2) biomass follows Bingham plastic behavior. The following observations were made during this research:

- Pressure oscillations were observed during injection of nutrient in porous media that had been inoculated with bacteria. The oscillations were characterized by growth and subsequent shearing of biomass from selected pore throats to form a breakthrough channel that was subsequently refilled.

- The first biomass plug developed at the nutrientinoculum interface. It formed as biomass grew along dominant flow pathways initially consisting of a series of interconnected, large pore throats. As these throats were filled, the dominant flow path shifted to the next series of smaller pore throats.

- Just before breakthrough, the first plug consisted of a number of empty pore throats interwoven throughout filled pore throats. These empty pores were found in both isolated interconnected groupings as well as individual empty pores.

- The breakthrough channel is initiated at the back boundary of the plug due to localized shearing in this area. The initiation of the breakthrough channel occurs at this point, because of the funneled nutrient flow to this area and the weaker biomass found there. The weaker biomass is a result of lower sucrose concentrations present in this region that produces less dextran that has a lower molecular weight, is less branched, and thus has a lower Bingham yield stress. The breakthrough channel initiates at the back of the channel and propagates backwards toward the front of the biomass plug (i.e., opposite the direction of nutrient flow).

- The first breakthrough channel in the first plug occurred when the normal pressure exceeded the Bingham yield stress of the biomass resulting in its being sheared from pore throats. This shearing produced a breakthrough channel by linking the sheared throats with the isolated groupings of empty pore throats and pore bodies that had been formed in the first plug. In this manner, a percolating backbone with nonconducting branches was formed.

- The location of the first breakthrough channel follows the series of interconnecting pore throats with the smallest wall surface area. Furthermore, the location of the breakthrough channel is indicated by a combination of the location of isolated pore throats in the plug and the location of an area of localized shearing at the back boundary of the plug.

- After formation of the first breakthrough channel, the channel began refilling with biomass almost immediately to form the second plug. After a period of time, other downstream pores also began to fill with biomass and the pressure drop increased to the point where the Bingham shear stress was again exceeded and a second breakthrough channel formed causing the pressure to decrease.

- The second breakthrough channel was more highly branched than the first breakthrough channel as a result of the higher fraction of empty pore throats in the second plug that had been incorporated into the channel. However, with subsequent plug develop- 
ment and breakthrough channel cycles, this area filled with biomass and became less branched.

- Biomass plugs with a high fraction of empty pore throats could be unstable, resulting in formation of breakthrough channels at a much lower value of maximum pressure drop. Whether or not the plug is stable depends on the location of biomass-filled pore throats, which is in turn dependent upon the pore-scale characteristics of the porous media, such as pore throat diameter.

- The pathways of subsequent backbones and branches changed with each plug propagation cycle. The change in location was attributed to in situ growth under higher shear stress conditions that produced stronger biomass than in adjacent pores.

\section{References}

Costerton JW. 1999. Introduction to biofilm. Int J Antimicrob Agents 2:217-221.

Costerton JW, Lewandowski Z, DeBeer D, Calldwell D, Korber D., James G. 1994. Minireview-Biofilms, the customized mirconiche. J Bacteriol 176:2137-2142.

Jack TR, DiBlasio E. 1985. Selective plugging for heavy oil recovery. In: Zajic JE, Donaldson EC, editors, Microbes and oil recovery. Vol. 1. El Paso, TX: Bioresources Publications. 205-212.

Jack TR, DiBlasio E, Thompson BG, Ward V. 1983. Bacterial systems for selective plugging in secondary oil production. Preprints, symposia, Vol. 27. Washington, DC: Division of Petroleum Chemistry, American Chemical Society, p 773-784.

Jack TR, Stehmeier LG. 1988. Selective plugging in watered out oil wells. In: Proceedings of the Symposium on Applications of Microorganisms to Petroleum Technology, Bartlesville, OK,
August 1987 (NIPER-351). Bartlesville, OK: National Institute for Petroleum \& Energy Research. p VII 1-13.

Jenneman GE, Knapp RM, Menize DE, McInerney MJ, Revus DE. 1984. Experimental studies of in-situ microbial enhanced oil recovery. Soc Petrol Eng Feb. 33-37.

Kim DS. 1999. Biomass evolution in porous media under bacterial starvation conditions. PhD thesis, University of Michigan, Ann Arbor, MI, USA.

Kim DS, Fogler HS. 2000. Biomass evolution in porous media and its effects on permeability under bacterial starvation conditions. Biotechnol Bioeng 69:47-56.

Lappan RE. 1994. Reduction of porous media permeability from in situ bacterial growth and polysaccharide production. $\mathrm{PhD}$ thesis, University of Michigan, Ann Arbor, MI, USA.

Lappan RE, Fogler HS. 1992. Effect of bacterial polysaccharide production on formation damage. SPE Prod Eng 7:167-171.

Lappan RE, Fogler HS. 1994. Leuconostoc mesenteroides growth kinetics with application to bacterial profile modification. Biotechnol Bioeng 43:865-873.

Lappan RE, Fogler HS. 1996. Reduction of porous media permeability from in situ Leuconostoc mesenteriodes growth and dextran production. Biotechnol Bioeng 50:6-15.

Powell MS, Slater NKH. 1982. Removal rates of bacterial cells from glass surfaces by fluid shear. 24:2527-2537.

Stewart TL, Fogler HS. 2001. Biomass plug development and propagation in porous media. Biotechnol Bioeng 72:353-363.

Stoodley PP, Lewandowski Z, Boyle JD, Lappin-Scott HM. 1999. Structural deformation of bacteria caused by short-term fluctuations in fluid shear: An in situ investigation of biofilm rheology. Biotechnol Bioeng 65:83-92.

Torbati HM, Raiders RA, Donaldson EC, McInerney MJ, Jenneman GE, Knapp RM. 1986. Effect of microbial growth on pore entrance size distribution in sandstone cores. J Indust Microbiol 1:227-234.

Wan J, Wilson JL, Kieft TL. 1994. Influence of the gas-water interface on transport of microorganisms through unsaturated porous media. App Environ Microbiol 60:509-516.

Wolf BF, Fogler HS. 2001. Alteration of the growth rate and lag time of Leuconostoc mesenteroides NRRL-B523. Biotechnol Bioeng 72:603-610. 